\title{
A Case of CFH-Ab Hus Responsive to Plasmapheresis and with Sustained Remission after Initiation of Immunosuppressive Therapy Highlighting the Common Pit Falls of a Complicated Disease
}

\section{Johannes Hofer ${ }^{1,2 *}$, Hannah-Sophie Arbeiter ${ }^{1}$, Mihály Józsi ${ }^{3}$, Thomas Giner ${ }^{1}$, Diana Dobiliene ${ }^{4}$, Jurate Masalskiene ${ }^{4}$, Jonas Surkus ${ }^{5}$ and Sarunas Rudaitis ${ }^{4}$}

${ }^{1}$ Department of Pediatrics, Innsbruck Medical University, Innsbruck, Austria

${ }^{2}$ Institute of Neurology of Senses and Language, Hospital of St John of God, Linz, Austria

${ }^{3}$ MTA-ELTE “Lendület" Complement Research Group, Department of Immunology, Eötvös Loránd University, Budapest, Hungary

${ }^{4}$ Clinic of Children Diseases, Hospital of Lithuanian University of Health Sciences Kaunas Clinics, Kaunas, Lithuania

${ }^{5}$ Department of Nephrology, Hospital of Lithuanian University of Health Sciences Kauno klinikos, Kaunas, Lithuania

${ }^{*}$ Corresponding author: Johannes Hofer, Innsbruck Medical University, Department of Pediatrics, Anichstrasse 35, 6020 Innsbruck, Austria, Tel: +4351250482459; E-mail: johannes.hofer@i-med.ac.at

Received date: May 07, 2016; Accepted date: May 18, 2016; Published date: May 20, 2016

Citation: Hofer J, Arbeiter HS, Józsi M, Giner T, Dobiliene D, et al. (2016) A Case of CFH-Ab Hus Responsive to Plasmapheresis and with Sustained Remission after Initiation of Immunosuppressive Therapy Highlighting the Common Pit Falls of a Complicated Disease. J Clin Exp Nephrol 1: 13. DOI: $10.21767 / 2472-5056.100013$

Copyright: (C) 2016 Hofer J, et al. This is an open-access article distributed under the terms of the Creative Commons Attribution License, which permits unrestricted use, distribution, and reproduction in any medium, provided the original author and source are credited.

Abstract

Background: Complement factor $\mathrm{H}$ antibody (CFH-Ab) associated hemolytic uremic syndrome (HUS) is different from the genetically defined aHUS forms, especially concerning diagnosis and treatment.

Case: We report a case of aHUS responsive to plasmapheresis with a slightly delayed detection of CFH-Ab after first disease recurrence. The initiation of immunosuppressive induction and maintenance therapy led to a sustained complete remission.

Conclusion: This case of CFH-Ab HUS responsive to PP and remission after initiation of IS supports the recently published guidelines on therapy of CFH-Ab aHUS and highlights the importance of a fast diagnosis of this rare disease entity.

Keywords: Complement Factor $\mathrm{H}$ antibodies; Antibody avidity; Atypical HUS; IVIG; Immunosuppressive therapy; MMF

\section{Abbreviations}

aHUS: Atypical HUS; AU: Arbitrary Units; CFH: Complement Factor $\mathrm{H}$; CFH-Ab: Complement Factor H Antibodies; C3: Complement Protein 3; HUS: Hemolytic Uremic Syndrome; IS: Immunosuppressant; IVIG: Intravenous Immunoglobulins; LDH: Lactate Dehydrogenase; MMF: Mycophenolate Mofetil; PP: Plasmapheresis

\section{Background}

Complement factor $\mathrm{H}$ antibody (CFH-Ab) associated hemolytic uremic syndrome (HUS) represents a distinct, antibody driven autoimmune form of atypical HUS [1]. The disease typically manifests in pediatric patients prior to puberty but it is also described in adult patients [2,3]. Early diagnosis and distinction from other forms of thrombotic microangiopathies is crucial as the antibody based dysregulation of the alternative pathway of complement implies antibody targeted therapy options: prompt induction of plasmapheresis (PP) and concomitant induction and maintenance immunosuppression (IS) is associated with a favourable outcome [2,4]. Eculizumab, a monoclonal antibody against $\mathrm{C} 5$, as alternative therapy option to PP, or in severe cases in combination with $\mathrm{PP}$, is actually also recommended but controversially discussed $[1,2]$. However, which IS agent to use is currently unclear.

We report a case of CFH-Ab HUS responsive to PP and with sustained remission after initiation of induction and maintenance IS therapy highlighting common pit falls in diagnosis and treatment of this orphan disease.

\section{Case Report}

In March 2011 a 13 year old girl presented with vomiting, oliguria, slight peripheral oedema and deterioration of health with severe weakness. Six days before an uncomplicated otitis media was diagnosed. Initial investigations in the pediatric clinic in Kaunas, Lithuania showed signs of microangiopathic hemolytic anemia, thrombocytopenia and acute renal impairment with decreased C3 and C4 (Table 1). Arterial blood pressure $(116 / 68 \mathrm{mmHg})$ was normal as was the neurologic 
status. The girl had no diarrhoea or neurological symptoms and ADAMTS 13 protease activity was normal. Atypical HUS was suspected so PP (as recommended in [5]) and hemodialysis were started within 24 hours after admission to the hospital. After 18 PP sessions total remission (normalization of hematologic parameters and renal function) was obtained. Seventeen days later PP had to be started again because of hematologic relapse (Hemoglobin $-5.7 \mathrm{~g} / \mathrm{dl}$, platelets $-87 \times 10^{3} / \mu \mathrm{l}$, haptoglobin -29 $\mathrm{mg} / \mathrm{l}, \mathrm{C} 3-48 \mathrm{mg} / \mathrm{dl}, \mathrm{LDH}-418 \mathrm{U} / \mathrm{l}$, serum creatinine $-1.07 \mathrm{mg} /$ dl). A total of 36 sessions till remission were necessary. At the time point of disease onset, but after initiation of PP serum samples for complement investigations were stored and 3 months after disease onset CFH-Ab HUS was diagnosed. Therefore, immune modulatory induction therapy with IVIG (2 $\mathrm{g} / \mathrm{kg}$ body weight on days 0,21 and 42) in addition to maintenance IS therapy with mycophenolate mofetil (started on day $2 ; 600 \mathrm{mg} / \mathrm{m}^{2}$ twice a day) was introduced. Seven months after disease onset one additional IVIG infusion was given as increasing $\mathrm{CFH}-\mathrm{Ab}$ titers were observed, although no clinical signs of relapse were noticed.

24 months after disease onset the patient is in sustained and complete remission.

Table 1: List of main laboratory parameter at disease onset and further laboratory investigations. ADAMTS13: a disintegrin and metalloproteinase with a thrombospondin type 1 motif member 13; CFHR: CFH-Related proteins; PP: Plasmapheresis.

\begin{tabular}{|c|c|}
\hline \multicolumn{2}{|c|}{ Initial Parameters } \\
\hline \multirow{3}{*}{ Hemolytic anemia } & Hemoglobin: $7.1 \mathrm{~g} / \mathrm{dl}$ \\
\hline & LDH: $2612 \mathrm{U} / \mathrm{I}$ \\
\hline & Haptoglobin: 86 mg/l \\
\hline Thrombocytopenia & Platelets: $55 \times 10^{3} / \mu \mathrm{l}$ \\
\hline Acute kidney injury & Creatinine: $13.79 \mathrm{mg} / \mathrm{dl}$ \\
\hline \multirow{2}{*}{$\begin{array}{l}\text { Initial complement } \\
\text { diagnostics }\end{array}$} & C3: $38 \mathrm{mg} / \mathrm{dl}(>80 \mathrm{mg} / \mathrm{dl})$ \\
\hline & C4: $9.6 \mathrm{mg} / \mathrm{dl}(>10 \mathrm{mg} / \mathrm{dl})$ \\
\hline \multicolumn{2}{|c|}{ Further laboratory investigations } \\
\hline \multirow{3}{*}{ TMA diagnostics } & ADAMTS 13 protease activity: $80 \%$ \\
\hline & $\begin{array}{l}\text { CFH-Ab titer after first PP sessions: } 400 \\
\mathrm{AU} / \mathrm{ml}\end{array}$ \\
\hline & Homozygous Deletion of CFHR1 \\
\hline
\end{tabular}

\section{CFH-Ab and CFH-Ab Avidity Testing}

$\mathrm{CFH}-\mathrm{Ab}$ titers were determined using an ELISA as previously published [6]. Briefly, ELISA plates were coated with purified human $\mathrm{CFH}$. Serum was added and detection was performed using goat antihuman IgG, labeled with horseradish peroxidase.

CFH-Ab avidity testing was performed as previously published [7]: ELISA plates coated with CFH were incubated with patient's serum. Thereafter incubation with $0.5 \mathrm{M}$ sodium thiocyanate (NaSCN), a chaotropic agent, and Tween ${ }^{\circledR}$-PBS was performed. The avidity index was calculated, by defining the ratio of remaining antibodies after $\mathrm{NaSCN}$ treatment and antibodies bound without NaSCN treatment.

\section{Discussion}

This case description points out some typical features and frequently seen problems in diagnosis and treatment of patients presenting with $\mathrm{CFH}-\mathrm{Ab}$ aHUS.

Initially, after prompt and correct diagnosis of aHUS, PP as first line therapy [5] in the pre-eculizumab era was introduced; however, the use of eculizumab would have been highly problematic due to economic reasons in the home country of that patient.

After fast and complete treatment response the decision to wean PP and monitor the further development of the patient was made. Due to economic reasons and the lack of availability of fast complement investigations in Lithuania no further diagnostic steps were taken at that time point. Those problems in diagnostics and treatment for highly specific orphan diseases are not unique for Lithuania but are the reality for a high number of patients, especially in developing regions and countries [8]. From this perspective, international, low or free charge cooperations mainly on a scientific basis are needed to facilitate proper diagnostic work-ups and even therapy in a stringent and cost-efficient way.

The early relapse developing 17 days after the last PP session in the presented patient is typical for CFH-Ab HUS treated without IS; around $80 \%$ of first disease relapses are observed within the first year and many of the patients without recurrence in the first year will not present a further acute disease episode thereafter $[1,2,6]$. Fortunately, the presented patient showed again prompt response to PP, which is often but not always the case for relapses of CFH-Ab HUS patients $[1,2,6]$. However, the relapse led to prompt initiation of detailed complement diagnostics via shipment of samples to a HUS research lab (Medical University Innsbruck, Department of Pediatrics I) as functional complement diagnostics within a scientific cooperation for free are often the only way for patients in similar countries to obtain proper complement diagnostic evaluation. Thus, the detection of CFH-Abs and the prompt initiation of an CFH-Ab targeted therapy using IVIG as induction and MMF as maintenance IS therapy was possible, leading to a sustained and complete remission in that patient. The current recommendations for CFH-Ab HUS therapy [2] are not far away from that approach but are further modified depending on the severity of extrarenal symptoms: therapy initiation with eculizumab or PP within the first two days after onset of aHUS. After early detection of CFH-Ab additional IS induction therapy based on the severity of extrarenal manifestations followed by a maintenance therapy with mycophenolate mofetil (MMF) and corticosteroids for at least one year should be initiated [1,2]. However, the kind of IS used as well as the decision if eculizumab and/or PP should be used remain an individual and empirical decision.

In contrast to the uncomplicated clinical course after initiation of MMF maintenance therapy disease monitoring was challenging as for most of those patients. Up to now frequent 
evaluation of $\mathrm{CFH}-\mathrm{Ab}$ titers in combination with markers of complement activation are recommended [1,2]. However, it is well known that on the one hand $\mathrm{CFH}-\mathrm{Ab}$ titers increase during phases of disease recurrence $[1,2,4,6]$, but on the other hand also increase without signs of disease activity especially during periods of uncomplicated infections [1,2]. Thus it is always challenging to make the correct therapeutic decision during phases of increasing CFH-Ab titers. With the knowledge that disease recurrence is mainly seen during the first year after disease onset one will be even more cautious in the case of increasing $\mathrm{CFH}-\mathrm{Ab}$ titers within that time period. In the presented case the necessary decisions during phases of increasing $\mathrm{CFH}-\mathrm{Ab}$ titers were further complicated due to the fact that no initial CFH-Ab titers before the initiation of PP were available. Thus, no real baseline CFH-Ab titer is available for that patient. That this dilemma is not unique to our patient is part of the daily clinical and scientific experience. Going through the literature on CFH-Ab HUS but also aHUS in general shows that this fact is demonstrated in many short comments on the reasons for the fragmentary data of many of those studies $[3,4,6,7]$. Thus, this case again demonstrates the outstanding importance of sample storage before therapy initiation of all patients presenting with symptoms consistent with thrombotic microangiopathies.

As shown in Figure $1 \mathrm{CFH}-\mathrm{Ab}$ avidity was measured for that patient retrospectively to document if this relatively new and not yet routinely used parameter could have served as additional monitoring parameter enabling an even more personalized and adequate therapeutic decision. Up to now there are only two studies dealing with the strength of binding between CFH-Abs and their epitopes [7,9]. Amongst them, only one is focusing on CFH-Ab HUS. Nozal et al. [7] performed a detailed analysis of avidity profiles in serial samples of $\mathrm{CFH}-\mathrm{Ab}$ positive aHUS patients: they found high inter-individual but only marginal intra-individual avidity differences leading to the conclusion that $\mathrm{CFH}-\mathrm{Ab}$ avidity together with $\mathrm{CFH}-\mathrm{Ab}$ titers and the clinical presentation of the patient could help to improve clinical decision making on an individualized basis. However, looking at the avidity course for our patient it was interesting to see that the avidity decreased after initiation of PP. Nevertheless, as initial avidity data before PP are missing no clear conclusion can be made. Furthermore, CFH-Ab avidity was highest during acute disease phase and directly prior to a CFH$\mathrm{Ab}$ titer increase during a febrile infection without additional signs of disease relapse.

Currently it is thought that a safe weaning of IS therapy should be taken into account not earlier than one year after its initiation for patients with uncomplicated diseases course (especially for those without central nerve system or cardiac system involvement), stable low CFH-Ab titers and no sign for ongoing complement activation [1,2]. However, as long as the IS therapy is well tolerated and without side effects the authors suggest that IS therapy should be weaned and stopped not earlier than two years after initiation due to several reasons: 1) Disease recurrence is mainly observed within the first and to some extent also during the second year after disease onset $[1,3,4] .2)$ If a patient remains without disease recurrence within the first year after disease onset recurrences thereafter are highly unlikely (personal observation; unpublished data in preparation for publication) 3) Despite the suggested good outcome for early diagnosed and adequately treated $\mathrm{CFH}-\mathrm{Ab}$ HUS patients it remains a quit heterogeneous disease with till now only little knowledge on long term progression and prognosis. Thus, the authors suggest a more careful and mainly individualized decision approach involving the caregivers in the decision process.

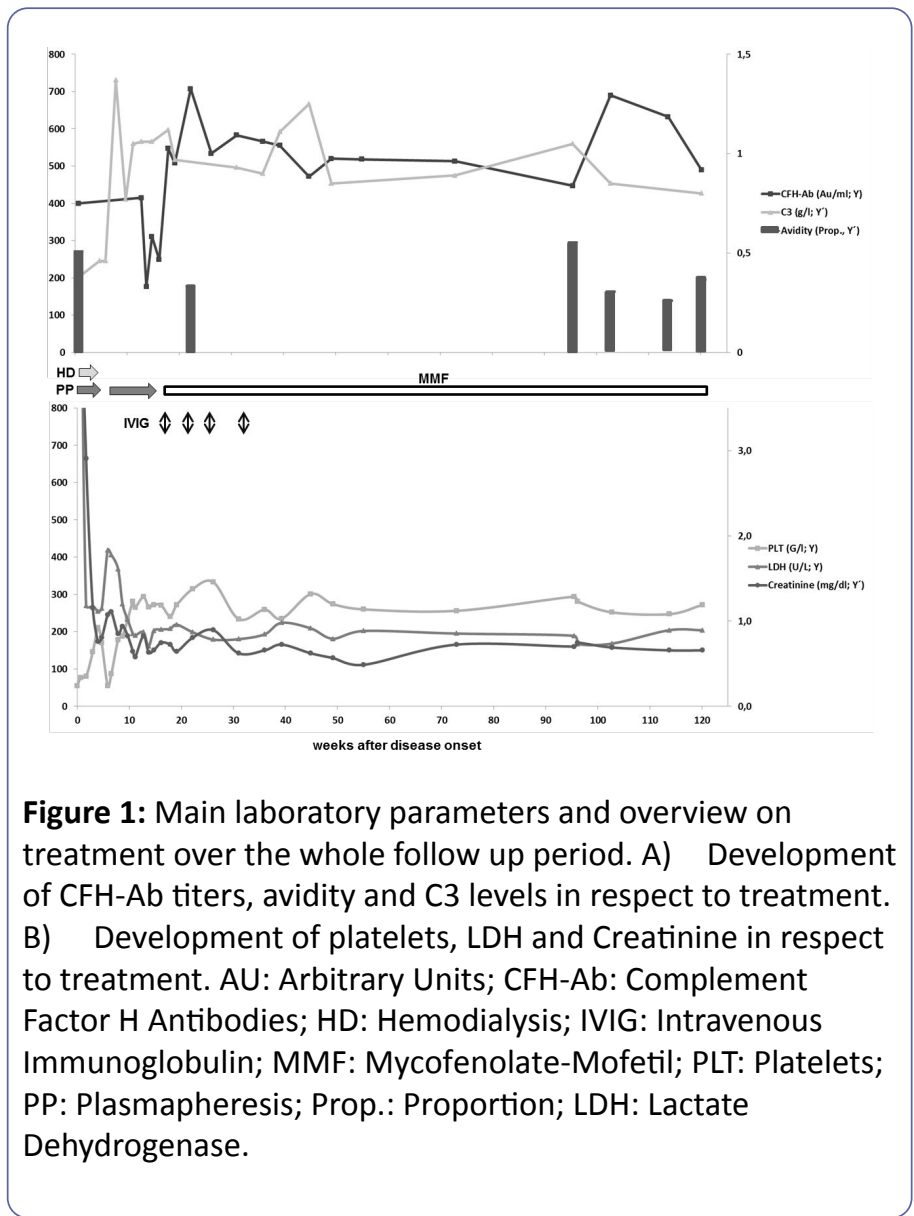

In conclusion, this case of CFH-Ab HUS responsive to PP with sustained remission after initiation of IS supports the recently published guidelines on the therapy of CFH-Ab HUS and highlights the importance of a fast diagnosis of this rare disease entity.

\section{Acknowledgements}

The work of J.H. is supported by the "Tiroler Wissenschaftsfond" and the "Österreichische Nationalbank" (Grant: 12711). M.J. is supported by the "Lendület" program of the Hungarian Academy of Sciences (grant LP2012-43).

\section{Conflict of interest}

J.H. received honorarium from Alexion Pharmaceuticals Inc. and served on advisory boards. 


\section{References:}

1. Hofer J, Giner T, Józsi M (2014) Complement Factor H-Antibodyassociated hemolytic uremic syndrome: pathogenesis, clinical presentation and treatment. Semin Thromb Hemost 40: 431-443.

2. Loirat C, Fakhouri F, Ariceta G, Besbas N, Bitzan M, et al. (2016) An international consensus approach to the management of atypical hemolytic uremic syndrome in children. Pediatr Nephrol 31: 15-39.

3. Dragon-Durey MA, Sethi SK, Bagga A (2010) Clinical features of anti-factor $H$ autoantibody-associated hemolytic uremic syndrome. J Am Soc Nephrol 21: 2180-2187.

4. Sinha A, Gulati A, Saini S (2014) Prompt plasma exchanges and immunosuppressive treatment improves the outcomes of antifactor $\mathrm{H}$ autoantibody-associated hemolytic uremic syndrome in children. Kidney Int 85: 1151-1160.

5. Ariceta G, Besbas N, Johnson S, Karpman D, Landau D, et al. (2009) Guideline for the investigation and initial therapy of darrhea- negative haemolytic uremic syndrome. Pediatr Nephrol 24: 687-696.

6. Hofer J, Janecke AR, ZimmerhackI LB (2013) Complement factor Hrelated protein 1 deficiency and factor $\mathrm{H}$ antibodies in pediatric patients with atypical hemolytic uremic syndrome. Clin J Am SocNephrol 8: 407-415.

7. Nozal P, Bernabéu-Herrero ME, Uzonyi B, Szilágyi Á, Hyvärinen S, et al. (2016) Heterogeneity but individual constancy of epitopes, isotypes and avidity of factor $\mathrm{H}$ autoantibodies in atypical hemolytic uremic syndrome. Mol Immunol 70: 47-55

8. Hofer J, Giner T, Safouh H (2014) Diagnosis and treatment of the hemolytic uremic syndrome disease spectrum in developing regions. Semin Thromb Hemost 40: 478-486.

9. Blanc C, Roumenina LT, Ashraf $Y$, Hyvärinen S, Sethi KS, et al. (2012) Overall Neutralization of Complement Factor $H$ by Autoantibodies in the Acute Phase of the Autoimmune Form of Atypical Hemolytic Uremic Syndrome 189: 3528-3537. 